\title{
About climate-seismicity coupling from correlation analysis
}

\author{
O. Molchanov \\ Institute of the Physics of the Earth, Russian Academy of Sciences, Bolshaya Gruzinskaya 10, 123995 Moscow, Russia \\ Received: 16 July 2009 - Accepted: 10 September 2009 - Published: 17 February 2010
}

\begin{abstract}
We have analyzed together the slow climate temperature variations in the near-equatorial Pacific Ocean area (SSTOI indices) and crustal seismic activity in the same region during 1973-2008 time period using correlation analysis and found similarity in seismic and ENSO periodicities (the latter with time lag about 1.5 years). Trends of the processes are also similar showing about 2 times increase in average seismic energy release during the whole period of analysis and conventional $0.1^{\circ} \mathrm{C} /(10$ years $)$ increase in SSTOI index anomalies. Our major conclusion is on real credibility of climate-seismicity coupling. It is rather probable that at least partially climate ENSO oscillations and temperature anomaly trends are induced by similar variation in seismicity.
\end{abstract}

\section{Introduction}

Topic of the slow climate changes is rather popular at present. Two important problems are usually discussed in connection with the climate variability: the several year quasiperiodicity (El Nino/La Nino or ENSO effect) and the longterm trend in the global temperature (global warming or cooling). Concerning forcing agent on the climate changes, several hypotheses have been suggested including changes in solar luminosity, variations in the Earth's orbit around the Sun, cosmic rays (e.g. Svensmark, 2007), volcanic eruption activity and so on but the most accepted cause is the change in atmospheric greenhouse gas concentrations due to human activity. At present the general consensus from data analysis and theoretical modeling is that global warming in the first half of 20th century can be attributed to either natural variation or human effects; however the warming since about 1975 is dominated by man-made greenhouse gas emissions. It seems that until now the climate coupling with the underground processes was discussed either in connection with volcano eruptions, which produce mainly cooling ef-

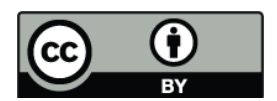

Correspondence to: $\mathrm{O}$. Molchanov (olmolchanov@mail.ru) fect (Robock and Oppenheimer, 2003), or in connection with tidal influence on seismic triggering, which revealed from analysis of short-term (about daily) and local seismic variations (e.g. Tsuruoka et al., 1995; Schekotov et al., 2006). Here we are going to analyze a correlation of the long-term and large-space variations in climate and earthquake activity.

\section{Data}

There are several methods to register the global climate changes and corresponding activity indices (see e.g. information of USA Climate Prediction Center http://www.cpc. ncep.noaa.gov/data/indices/): zonal and trade wind indices, sea level pressure indices (SOI), warm water volume indices (WWV), outgoing long wave radiation indices and at last sea surface temperature indices (SSTOI). We select the latter indices, which are probably the most reliable and suitable for us and which were registered without interruptions since 1950 until the present time in the four zones shown in Fig. 1.

First of all we calculate the climate index differences:

$$
d T(i)=\operatorname{SSTOI}(\text { zone } i)-\langle\text { SSTOI }(\text { zone } i)\rangle
$$

$i=1,3,4,34$ (see nominations in Fig. 1), where averaging is over all the period of analysis and SSTOI indices are taken in the time interval $\Delta t=6$ months. The temperature differences are measured in ${ }^{\circ} \mathrm{C}$. Then we produce smoothing of the 6-months data by the 1-year running window in order to avoid seasonal variations and obtain smoothed indices $d T_{s}(i)$. Further, we smooth the values $d T s(i)$ by 20 -years running window in order to find the trends $\operatorname{tr} d T(i)$ and compute the detrended variation: $d T t(i)=d T s(i)-t r d T(i)$. Concerning the earthquake (EQ hereafter) data, we use USGS catalog and compute a cumulative seismic energy release $E_{\mathrm{S}_{\Sigma}}=\Sigma E_{\mathrm{S}_{j}}$ in each 6-month interval for each of selected zones by using conventional relation for individual EQ with seismic energy $E_{\mathrm{S}}$ (Kanamori and Anderson, 1975):

$\log \left(E_{\mathrm{S}}\right)=1.5 M+4.8$

where $M$ is EQ magnitude. Then we introduce the indices of 


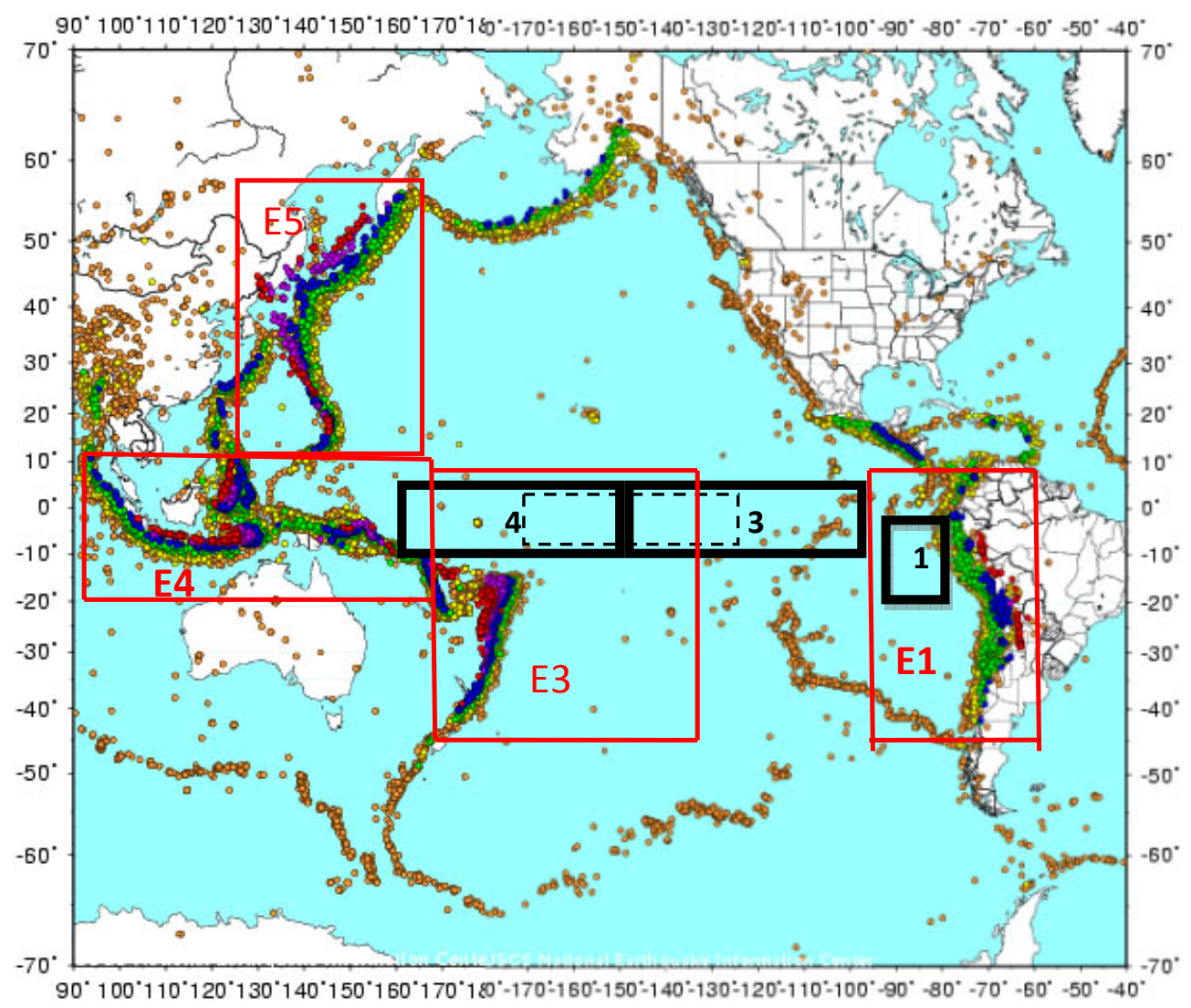

Fig. 1. Map of data collection. Thick rectangles show zones of SSTOI indices registration: Nino 1+2 (0-10S, 90-80W), Nino 3 (5N-5S, 150-90W), and Nino 4 (5N-5S, 160E-150W). Zone of Nino 3.4 (5N-5S, 170-120W) is shown by dashed line rectangle. Selected zones of earthquake activity are shown by solid line rectangles: E1 (Chilean subduction zone), E3 (Tonga-Kermadec zone), E4 (Sunda, Philippine, Solomon Sea zones), and E5 (Mariana, Japan and Kurile-Kamchatka zones). Distribution of EQ epicenters with depth $d>500 \mathrm{~km}$ and magnitude $M>5$ is shown by red points, those with depth $<33 \mathrm{~km}$ are shown by yellow points, and EQ epicenters with intermediate depth are shown by green, brown and blue points (from USGS catalog).

seismic variability as following:

$e(t)=\log \left(E_{\mathrm{S}_{\Sigma}}\right)-\left\langle\log \left(E_{\mathrm{S}_{\Sigma}}\right)\right\rangle$

In such a way we follow to Richter's definition of regional seismic activity index $a(t)$, involved in the famous Gutenberg-Richter law of EQ number distribution against the magnitude:

$\log [N(M \geq M \pm)]=a-b M \pm$

where $b \approx 0.9$ is the constant connected with the fractal distribution of EQs in space. By using relations (1) and (3), we can easily show:

$\log \left(E_{\mathrm{S}_{\Sigma}}\right)=(1.5 / b) a+c$

where

$c=4.8+\log \left[\left(1-N_{j}^{-p}\right) / p\right]$ and $e(t)=1.5 / b(a-\langle a\rangle)$

provided that $N_{j} \gg 1$. Here parameter $p=(1.5-b) / b \approx 0.7$ and $N_{j}$ is the number of the strongest EQs in the time cell
$\Delta t$. We include into analysis several thousands of the largest crustal EQs (with depths ranging from 0 to $38 \mathrm{~km}$ ) within each zone during the whole selected time period from 1973 to 2008 ( $\mathrm{Ta}=35$ years). Some statistical characteristics of seismic activity involved in our analysis are presented in Table 1.

As envisaged from the Table 1 we compute the energy release and corresponding values $e(n)$ in each n-zone $(n=1$, $3,4)$ and in addition the indices $e(34)$ and $e($ eq) in the aggregate region $\mathrm{E} 34(\mathrm{E} 3+\mathrm{E} 4)$ and in the near-equatorial area $\mathrm{E}(\mathrm{eq})=\mathrm{E} 34+\mathrm{E} 1$. Similarly to climate indices we apply the 1 -year smoothing, find the trends, and detrend the original variations. Also, we calculate the seismic indices for deep EQs $e$ (nd) (depth $>300 \mathrm{~km}$, the reason for doing this is explained later). Examples of the indices behavior are presented in Fig. 2. It can be seen that climate indices show expected ENSO variation. Amazingly, but EQ indices demonstrate the similar quasi-ENSO variations. Even from these examples we note the main features of the presented results: a) $d T t(i)$ curves are almost coincident (we compare in Fig. 2a the averged over four SSTOI zones index $d T t(\mathrm{av})$ and $d \operatorname{Tt}(34)$ 
Table 1. Averaged values of regional seismic energy release $\langle E\rangle$ and averaged number of strongest EQs $\langle N j\rangle$ in the time interval of 6 months.

\begin{tabular}{lcccccc}
\hline Energy/Zones & E1 & E3 & E4 & E5 & E34 & E(eq) \\
\hline$\langle E\rangle$ & & & & & & \\
(in crust), & 107 & 251 & 940 & 222 & 1191 & 1299 \\
$10^{14} \mathrm{~J} / 6$ months & & & & & & \\
$N$, (Number of & & & & & & \\
EQ in crust); & 2832 & 5560 & 8104 & 7094 & 13664 & 16496 \\
$\langle N j\rangle$ & 40 & 79 & 115 & 101 & 195 & 235 \\
$\langle E\rangle$ & & & & & & \\
(in depth $>300 \mathrm{~km})$, & 34.4 & 29.9 & 19.4 & 14 & 49.3 & 83.7 \\
$10^{14} \mathrm{~J} / 6$ months & & & & & & \\
$N($ depth $300 \mathrm{~km}) ;$ & 277 & 3540 & 2236 & 521 & 5776 & 6053 \\
$\langle N j\rangle$ & 4 & 50 & 32 & 7 & 82 & 86 \\
\hline
\end{tabular}

in the central zone). It means rather large spatial variation size covering the whole near-equatorial Pacific area. Therefore, we consider in what follows only $d T t(\mathrm{eq})$ and $d T t(34)$ values. Unlike them, the $\operatorname{et}(n)$ curves deviate from zone to zone, but an increase of zone size improves the similarity with the $d T t(i)$ behavior; b) visually, the fitting of the $d T t(\mathrm{eq})$ and et (eq) curves is the best when the seismic curve is shifted in time by about 1.5 year ahead.

Let us test the above conclusions with the formal correlation analysis.

\section{Correlation analysis}

The correlation analysis is a rather powerful tool to reveal the periodicities hidden in the given processes. For example, if a process consists of the random phase signal $A_{0}(t)$ and an oscillation of the period of $T_{1}$, the following formula is valid:

$x(t)=A_{0}(t)+A_{1} \cos \left(2 \pi t / T_{1}\right)$

It is easy to find the auto-correlation function of process (5) as follows:

$$
\begin{aligned}
R_{x x}(\tau) \equiv & x \pm x \approx\left[<A_{0}^{2}>\exp \left\{-\tau^{2} / \Delta t^{2}\right\}\right. \\
& \left.+A_{1}^{2} \cos \left(2 \pi \tau / T_{1}\right)\right] /\left[<A_{0}^{2}>+A_{1}^{2}\right]
\end{aligned}
$$

where $\Delta t$ is the average time of phase transfer or the sampling time in digitized signal and it is supposed that $\Delta t \ll T a, T_{1}$. It is obvious that $R_{x x}=1$ and there are maxima at shifts $\tau_{m}= \pm k T_{1},(k=1,2,3, \ldots)$ each having the relative amplitude of $A_{1}^{2} /\left[<A_{0}^{2}>+A_{1}^{2}\right]$. Autocorrelations of the ENSO temperature variation and the seismic activity variation in the same regions as in Fig. 2 are shown in Fig. 3a and b. Both autocorrelations show similar $4.5-5$ years periodicity. An important question arises immediately: do climate changes induce the seismic variations (the first variant of explanation)? Or on contrary, the seismic variations generate the climate ENSO changes (the second variant)? This is the

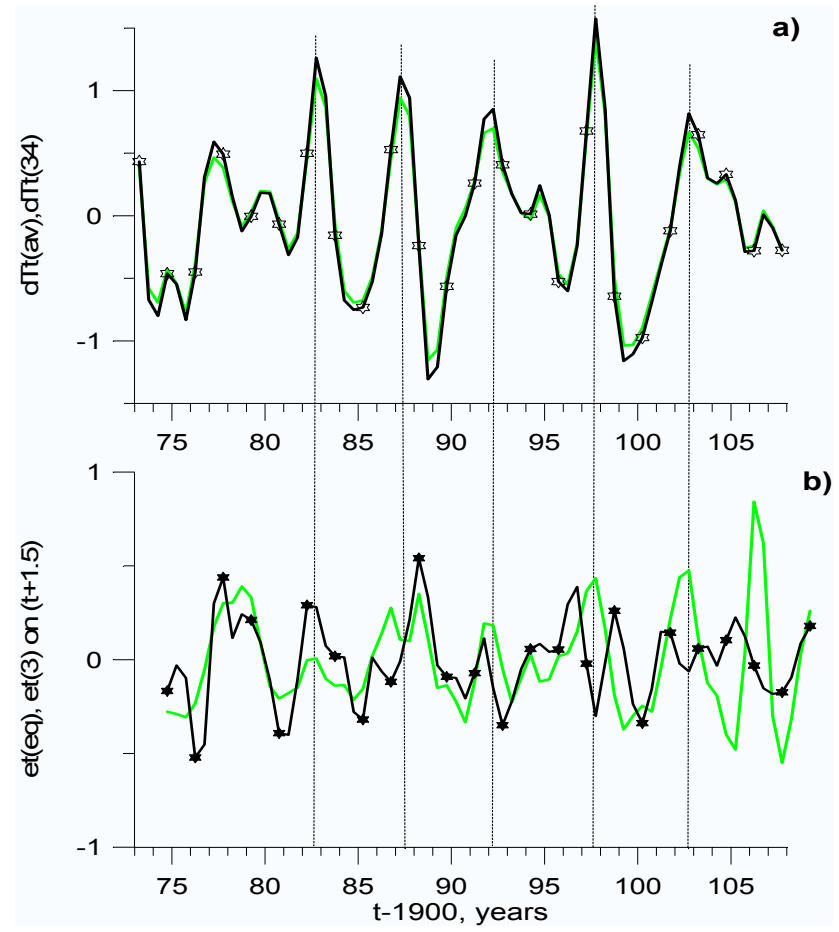

Fig. 2. (a) Detrended temperature variation in the near-equatorial area of the Pacific Ocean (solid line) and in the cenral zone of the Ocean (line with open circles); (b) Detrended seismic activity variation in the area E(eq) (solid line) and in the E34 (line with filled circles) shifted in time by 1.5 years ahead. For convinience the main El Nino maxima are indicated by vertical dash lines.

so-called mother-daughter (egg-hen) problem. One approach to resolve this proplem is the comparison of seismic periodicity in the crust and in the depth. In case when the periodicity decreases with depth then the first variant is valid. But when the periodicity does not disapper with depth, then the second explanation is more probable. However, such an approach is hampered by different relative level of seismic activity at the depth and in the crust. Anyway, we present the comparison of the crust-depth periodicity in zone E4 (Fig. 3c), where the crust activity is maximal but the deep seismic activity is not so high (see Table 1), and in the zone E1, where on contrary, the activity in depth is maximal but the crustal activity is low (Fig. 3d). In both cases the periodicity is a little varied but its relative amplitude is about the same.

The second approach to resolve our mother-daughter problem is application of the cross-correlation analysis. If in additon to $x(t)$ described by relation (6), there is the other variation $y(t)=B_{0}(t)+B_{1} \cos \left[2 \pi\left(t-\tau_{d}\right) / T_{1}\right]$, which contains similar periodic component shifted in phase, then their crosscorrelation is as follows:

$x \pm y=A_{1} B_{1} \cos \left[2 \pi\left(\tau-\tau_{d}\right) / T_{1}\right] / \sqrt{\left[\left(\left\langle A_{0}^{2}\right\rangle+A_{1}^{2}\right)\left(\left\langle B_{0}^{2}\right\rangle+B_{1}^{2}\right)\right]}$

In this example, there are maxima at $\tau_{m}=\tau_{d} \pm k T_{1}$. The crosscorrelation of the climate temperature and the seismic crustal 


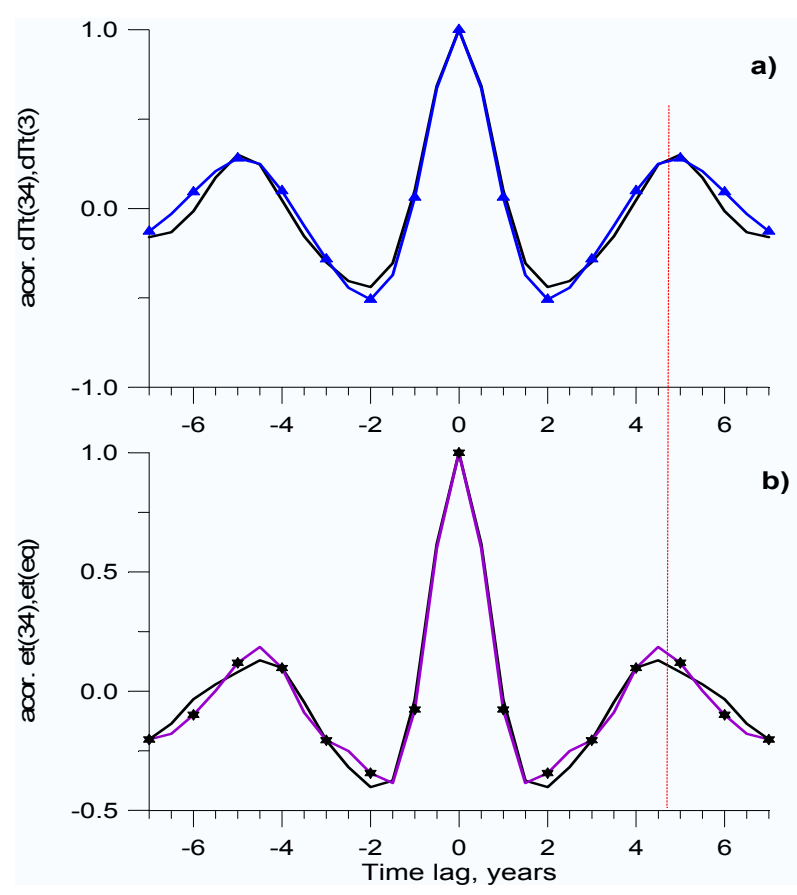

a)

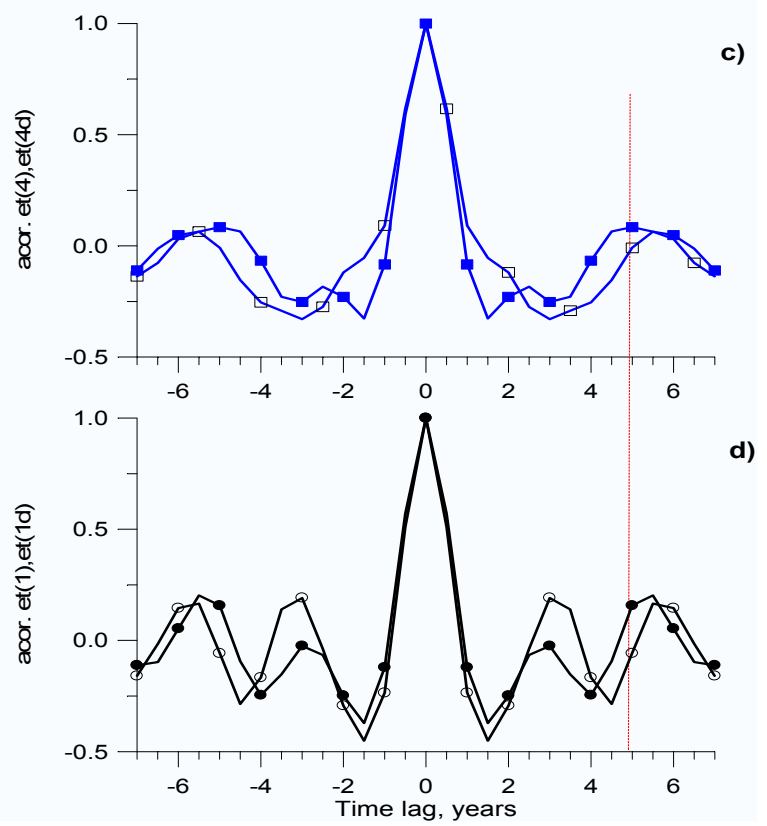

Fig. 3. (a) Autocorrelation of temperature variations in zone 34 (solid line) and zone 3 (triangles); (b) Autocorrelation of seismic variations in the seismic zones E(eq) (solid line) and E34 (stars); (c) Comparison of autocorrelation of seismic activity variation in crust (filled squares) and in the depth (open squares) for the seismic zone E4; (d) Comparison of autocorrelation in crust(filled circles) and in depth (open circles) for zone E1. Vertical dash line shows averaged ENSO period.

activity variation is shown in Fig. 4a. Interpretation of the results in Fig. 4a is not simple. From one hand, it supports the conclusion on common periodicity of the climate and seismic variations. From the other hand, it does not help clarifying their causative relation: the maximum at $\tau_{d 1} \approx+1.5$ years could correspond to the climate variation that follows the seismic induction, while the maximum at $\tau_{d 2} \approx-3.5$ years means the climate impact on the seismicity. We can assume two possible scenarios:

- a) A pulse of the climate perturbation (or a maximum in its variation) at the time $t_{i}$ appears in the crust at time $t_{c}$ and then penetrates in the some deep layer of the Earth at time $t_{0}$. In this case $t_{i}-t_{c}=\tau_{d 2}$ and $t_{i}-t_{0}=\tau_{02}<\tau_{d 2}<0$. It corresponds to the possibility of climate influence on the EQ triggering.

- b) A pulse of perturbation is originated in the depth at $t_{0}$ and then it reaches the crust and after it appears as climate temperature increase with the time delay $\tau_{d 1}$. In this case: $t_{0}-t_{i}=\tau_{01}>\tau_{d 1}>0$. It corresponds to the hypothesis of the EQ activity forcing the ENSO variation in the climate.

Recently, possibility of upward migration of the EQ hypocenters was discussed in the papers by Molchanov and Akentieva (2008) and Molchanov and Uyeda (2008). The statistical study was presented on the EQ activity in the 5 layers from mantle $(d=500-700 \mathrm{~km})$ to the crust $(d \leq 38 \mathrm{~km})$, the technique of so-called multi-layer correlation was used that showed the time delay of EQ activity progression between 2 and 10 years for different subduction zones. Here, we use a simplified version of the same technique by computing the triple correlation: $\operatorname{et}(\mathrm{nd}) \pm \operatorname{et}(n) \pm d T t(i)$. The triple correlation function for zones E4 and E1 is presented in Fig. 4b. Prevailing of maxima with $\tau>0$ means that scenario $b$ ) is more probable than scenario a).

\section{Trends}

Trends of the climate and EQ variations are presented in Fig. 5. Progress with time in the climate indices $0.1^{\circ} \mathrm{C} /(10$ years $)$ is a conventional value for the ocean surface, where the temperature changes are about two times smaller than above the ground (Smith et al., 2005). The seismic indices show about 2 times increase in the average seismic energy release both in the crust and in the depth beginning from 1983.

\section{Discussion and conclusions}

Our major conclusion is on a real possibility of the climateseismicity coupling. It is rather probable that the climate ENSO effect is at least partially induced by seismicity with 

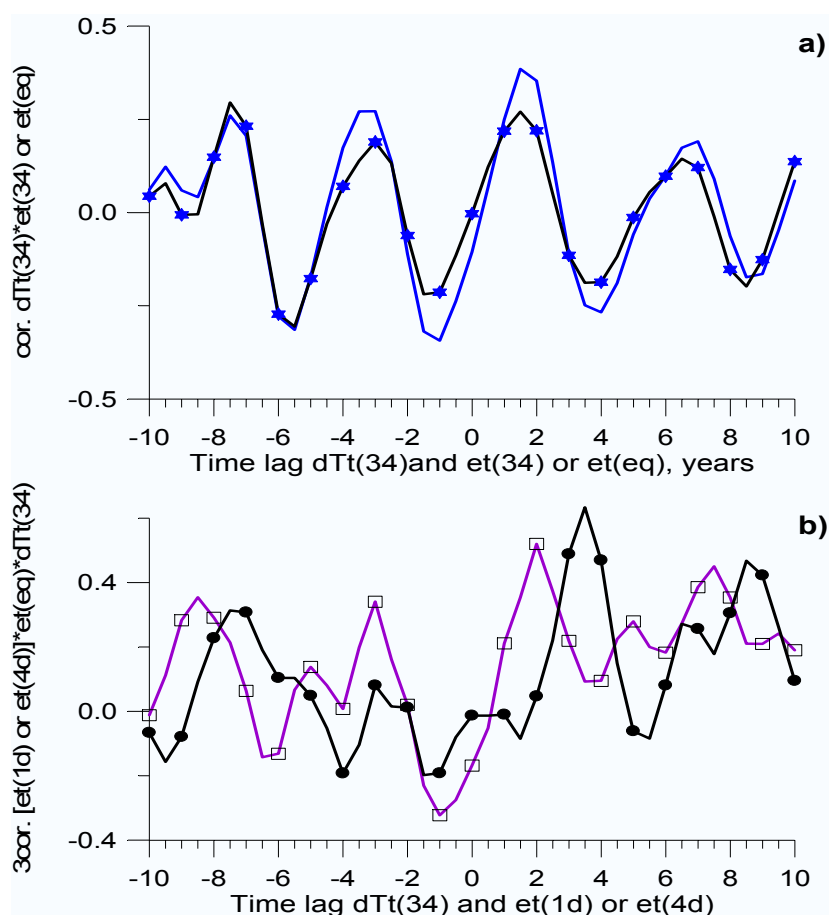

Fig. 4. (a) Cross-correlation of temperature and seismic variation in zone E(eq) (solid line) and in zone E34 (line with stars); (b) Triple correlation $\operatorname{et}(1 \mathrm{~d}) \pm \operatorname{et}(\mathrm{eq}) \pm d T t(34)$ (filled circles) and et $(4 \mathrm{~d}) \pm e t(\mathrm{eq}) \pm d T t(34)$ (squares).

a time lag of about 1.5 years. Trends in the climate and seismic variations are similar to each other. Possibility of the seismic influence on the climate seems surprising especially when taking into account a conventional consideration of the Earth heat budget. Major source of heat is the solar radiation, which provides power flux at atmosphere $P_{\mathrm{SR}}=340 \mathrm{~W} / \mathrm{m}^{2}$. This factor heats the Earth to temperature $T_{0} \approx 288^{\circ} \mathrm{K}$. At the first sight the EQ source can be considered as a negligible one. As it is evident from Table $1\langle E\rangle \approx 1.5 \times 10^{17} \mathrm{~J}$ in the Pacific region having the area $S_{O} \sim 10^{13} \mathrm{~m}^{2}$, the average power flux is $\left\langle P_{\mathrm{SE}}\right\rangle=\langle E\rangle /\left(S_{O} \Delta t\right) \approx 0.001 \mathrm{~W} / \mathrm{m}^{2}$, where as before $\Delta t=6$ months $\approx 1.5 \times 10^{7} \mathrm{~s}$. However, the seismic energy is only a small part of mechanical work $W_{\mathrm{E}}$ done in the earthquake faulting. Their relation is the following:

$E=K_{\mathrm{SE}} W_{\mathrm{E}}$

where $K_{\mathrm{SE}} \sim \Delta \sigma / \sigma$ is the seismic efficiency, $\sigma$ is the mean shear stress acting on a fault and $\Delta \sigma$ is the stress-drop. Taking into consideration the usual values of $\Delta \sigma \sim 0.3-1 \mathrm{MPa}$ and $\sigma \sim 300-500 \mathrm{MPa}$, we obtain $K_{\mathrm{SE}} \sim 0.001-0.003$. A transient heat flux is generated during coseismic slip as a conversion of the mechanical work done in faulting. Because of low thermal conductivity, this huge heat flux leads to local heating or even vaporization of fluids and its upward migration. Supposing that all the heat produced by EQs reaches the ground surface (the ocean bottom), we have the other es-

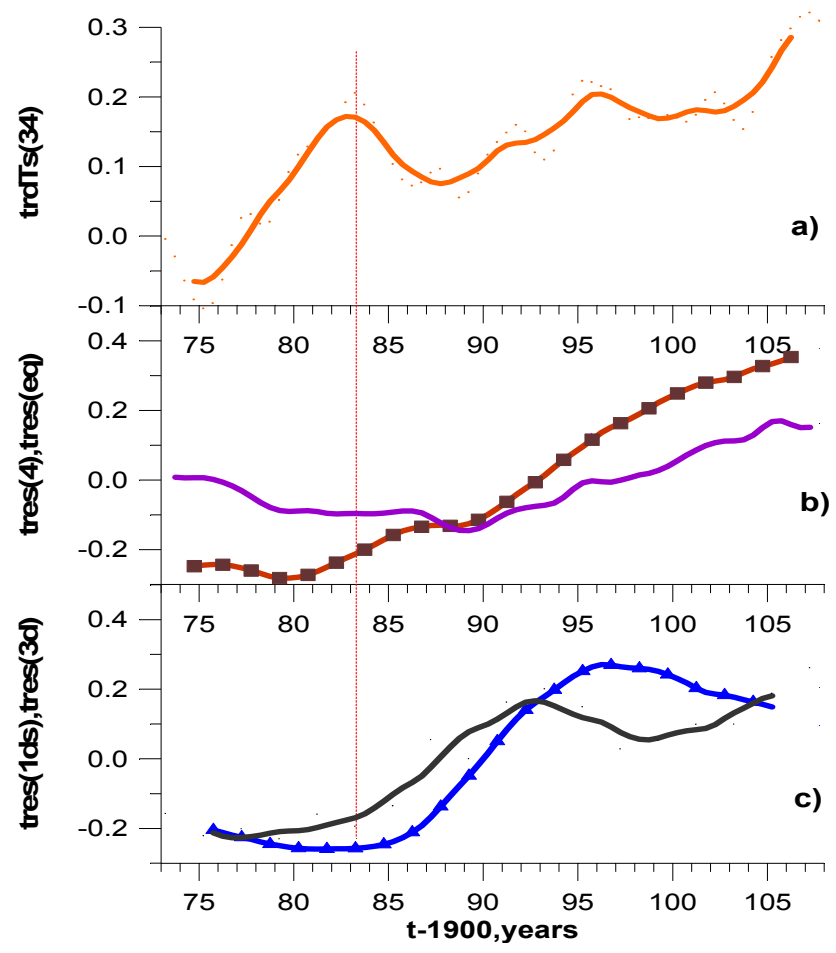

Fig. 5. (a) Trend of the SSTOI (34) variation; (b) Trends of the seismic activity in the crust, zone E4 (squares) and equatorial zone (line); (c) Trends of the seismic activity in the depth $>300 \mathrm{~km}$, in zone E1 (line) and in zone E3 (triangles).

timate for the average power flux due to seismicity: $\left\langle P_{\mathrm{E}}\right\rangle=$ $\left\langle P_{\mathrm{SE}}\right\rangle / K_{\mathrm{SE}} \approx 0.3-1 \mathrm{~W} / \mathrm{m}^{2}$. Let us estimate now its ability to induce the ENSO variation. Rough estimation using StefanoBoltzmann law for black body irradiance is as follows:

$$
\begin{aligned}
\delta T & =\Delta T\left(t_{2}\right)-\Delta T\left(t_{1}\right) \\
& =T_{0} /\left(4 P_{\mathrm{SR}}\right)\left[P_{\mathrm{E}}\left(t_{2}\right)-P_{\mathrm{E}}\left(t_{1}\right)\right] \\
& \approx 0.21\left[P_{\mathrm{E}}\left(t_{2}\right)-P_{\mathrm{E}}\left(t_{1}\right)\right]
\end{aligned}
$$

where $\Delta T$ is deviation of $T$ from background level $T_{0}$ and $t_{2}, t_{1}$ is ending and starting time of recording. Let us recall that seismic index $e(t) \approx \log 10(E /\langle E\rangle)$, hence its variation from -0.5 to +0.7 (see Fig. $2 b$ ) corresponds to the Evariation from $0.3\langle E\rangle$ to $5\langle E\rangle$, which leads us to estimate of $\delta T_{\mathrm{E}} \approx 0.3-0.9^{\circ} \mathrm{C}$. The value is comparable but eventually smaller than the scope of ENSO variation $\sim 2{ }^{\circ} \mathrm{C}$ (Fig. 2a). Application of relation (9) to analysis of trends leads to estimate $\delta T_{\mathrm{E} t}$ (change in trend from 1983$) \approx 0.06-0.4^{\circ} \mathrm{C}$ that is also comparable with the change in ocean temperature (see Fig. 5a). Acceleration of the greenhouse rate in the postindustrial era and the consequent global warming is considered now as an urgent social hazard (e.g. Hegerl et al., 2007). ENSO oscillations could appear within this concept as a result of various feedbacks in the energy exchange between the ground and troposphere including the water evaporation, changes in the lapse rate, ice-albedo feedback and 
so on (e.g. Soden and Held, 2005). On the other hand, the earthquake influence could be also rather important, as our results and estimates show. It is appropriate to mention here that EQ energy release in the near-equatorial Pacific area during the half a year period is $\approx 10^{20} \mathrm{~J}$, which is equivalent to the energy released in the detonation of about million atomic bombs of Hiroshima class (13 kT TNT).

Acknowledgements. I am grateful to Olga Akentieva and Andrew Tronin for their help and useful discussions.

Edited by: M. E. Contadakis

Reviewed by: A. P. Nickolaenko and another anonymous referee

\section{References}

Hegerl, G. C., Zwiers, F. W., Braconnot, P., et al.: Understanding and Attributing Climate Change, in: Climate Change 2007: The Physical Science Basis, Contribution of Working Group I to the Fourth Assessment Report of the Intergovernmental Panel on Climate Change, Cambridge Univ. Press, ch. 9, 1-14, 2007.

Kanamori, H. and Anderson, D.: Theoretical basis of some empirical relations in seismology, B. Seismol. Soc. Am., 65, 1073-95, 1975.
Molchanov, O. A. and Aken'tieva, O. S.: Upward Motion of Earthquake Hypocenters in the Wadati-Benioff Seismic Zones, Russian Academy Reports, 419, 473-476, 2008.

Molchanov, O. A. and Uyeda, S.: Upward migration of earthquake hypocenters in Japan, Kurile-Kamchatka and Sunda subduction zones, Phys. Chem. Earth, 34, 423-430, 2009.

Robock, A. and Oppenheimer, C. (Eds.): Volcanism and the Earth's Atmosphere, American Geophysical Union, Washington, DC, Geophysical Monograph, 139, 360 pp., 2003.

Schekotov, A. Yu., Molchanov, O. A., and Hayakawa, M.: A study of atmospheric influence from earthquake statistics, Phys. Chem. Earth, 31, 341-345, 2006.

Smith, T. M. and Reynolds, R. W.: A Global Merged Land-Air-Sea Surface Temperature Reconstruction Based on Historical Observations (1880-1997), J. Climate, 18(12), 2021-2036, 2005.

Soden, B. J. and Held, I. M.: An Assessment of Climate Feedbacks in Coupled Ocean-Atmosphere Models, J. Climate, 19(14), 3354-3360, 2005.

Tsuruoka, H., Ohtake, M., and Sato, H.: Statistical test of the tidal triggering of earthquakes: contribution of the ocean tide loading effect, Geophys. J. Int., 122, 183-194, 1995. 